\title{
Psicooncología
}

ISSN: 1696-7240

http://dx.doi.org/10.5209/psic.72023

\section{Curar al alumno, no solo su enfermedad. Descripción de un caso}

\author{
Sergio Yáñez Cañas ${ }^{1}$; Marta Fierros Sánchez-Cuenca²; Camino Bengoechea Menéndez; \\ Blanca López-Ibor Aliño, ${ }^{4 *}$
}

Recibido: 17 de junio de 2020 / 10 de agosto de 2020

Resumen: Introducción: En los últimos años las aulas hospitalarias se han configurado como un elemento de gran importancia en la vida de los niños y adolescentes que se encuentran en tratamiento de diferentes enfermedades. Los pacientes de enfermedades oncológicas no son una excepción. Objetivo: Aunque las aulas hospitalarias en la actualidad son capaces de sincronizar su trabajo con los colegios de referencia de los pacientes, el propósito de este artículo es presentar y describir un estudio de caso que subraya el papel del aula hospitalaria en sí misma, como una herramienta educativa que es útil en la tarea de educar a sus alumnos de una forma global e inclusiva, sin soslayar los niveles de exigencia adecuados. Método: Presenta el caso de un adolescente de 16 años diagnosticado de un osteosarcoma localizado que requirió quimioterapia preoperatoria y postoperatoria, cirugía de alta complejidad y finalmente un tratamiento inmunomodulador por un tiempo prolongado. No tenía ningún tipo de actividad académica al haber terminado la etapa de aprendizaje obligatorio y no estaba matriculado en ningún colegio, y a través del Aula Hospitalaria de la Unidad de Hematología y Oncología Pediátrica del Hospital HM Montepríncipe llegó a ser un estudiante interesado y motivado. A pesar de que su enfoque es necesariamente multidisciplinar, este estudio presenta una perspectiva educativa y pone de manifiesto la función, posibilidades, ventajas y desventajas de las aulas hospitalarias y su coordinación con el resto del personal. Lo hace poniendo al paciente en medio de su enfoque, a través de una visión humanística que enfatiza el verdadero significado de la educación. Conclusiones: El propósito de un aula hospitalaria no debe ser solo académico, pues tiene un papel fundamental en el objetivo de que el niño y adolescente con cáncer se convierta en un adulto sano física, psíquica, social y espiritualmente. Palabras clave: Oncopediatría, cáncer infantil, cáncer del adolescente, aula hospitalaria.

1 Sergio Yáñez Cañas. Aula Hospitalaria Colegio Montepríncipe, Unidad de Hematología y Oncología Pediátrica, Hospital Universitario HM Montepríncipe.

E-mail: syanez@hmhospitales.com

2 Marta Fierros Sánchez-Cuenca. Aula Hospitalaria Colegio Montepríncipe, Unidad de Hematología y Oncología Pediátrica, Hospital Universitario HM Montepríncipe.

E-mail: mfierros@hmhospitales.com

3 Camino Bengoechea Menéndez. Unidad de Hematología y Oncología Pediátrica, Hospital Universitario HM Montepríncipe.

E-mail: cbengoechea@hmhospitales.com

4 Blanca López-Ibor Aliño. Unidad de Hematología y Oncología Pediátrica, Hospital Universitario HM Montepríncipe.

E-mail: blopezibor@hmhospitales.com

* Dirección de correspondencia: Sergio Yáñez Cañas. Aula Hospitalaria Colegio Montepríncipe. Unidad de Hematología y Oncología Pediátrica del Hospital Universitario HM Montepríncipe. Avenida de Montepríncipe 25, 28660, Boadilla del Monte (Madrid)-E-mail: syanez@hmhospitales.com 


\title{
[en] Healing a student, not just his or her disease. A Case description
}

\begin{abstract}
Introduction: The importance of hospital schools has been rising during the last decades. They have gained a lot of influence in the lives of children and teenagers under treatment for different diseases. Those in pediatric hematology/oncology units are not an exception. Objective: Though the hospital schools are nowadays capable of synchronize their work with the patients' regular schools, the purpose of this project is to present and describe a case report which underlines the role of the hospital schools as themselves, as a teaching tool which is useful in the goal of educating its students in a global and comprehensive way, which includes an adequate level of demand. Method: It presents the case of a 16 years old boy diagnosed with a localized osteosarcoma that required preoperative and post-operative chemotherapy, high complexity surgery and, finally, immunomodulatory therapy for a long time. $\mathrm{He}$ had not any kind of learning activity, as he had finished the obligatory educational stage and was not enrolled in any school, and through the Hospital School in the Pediatric Hematology/Oncology Unit in the HM Montepríncipe Hospital became an interested and motivated student. Though its approach is necessarily cross-disciplinary, the present report has an educational perspective and it stresses the function, possibilities, advantages and disadvantages of hospital schools within pediatric oncology units and their coordination with the rest of the staff. It does it by putting the patient in the middle of its scope, through a humanist vision that emphasizes the real meaning of education. Results: The purpose of hospital schools should not be just academic, since they have a fundamental role in achieving that children and teenagers with cancer become healthy adults in a physic, psychic, social and spiritual way. Keywords: Pediatric oncology, children's cancer, cancer in adolescents, hospital school.
\end{abstract}

Sumario: 1. Introducción 2. El caso 2.1. Primer trimestre 2.2. Segundo trimestre 2.3. Tercer trimestre 2.4. Cuarto trimestre 3. Discusión 5. Conclusiones 6. Referencias bibliográficas

Cómo citar: Yáñez Cañas S, Fierros Sánchez-Cuenca M, Bengoechea Menéndez C, López-Ibor Aliño B. Curar al alumno, no solo su enfermedad. Descripción de un caso. Psicooncología 2020; 17: 375-385. doi: $10.5209 /$ psic.70199

\section{Introducción}

La aparición y puesta en funcionamiento de las aulas hospitalarias en España es un fenómeno relativamente reciente, por lo queda un largo camino en lo que respecta a su definición y perfeccionamiento.

En efecto, aunque se habían llevado a cabo experiencias de educación hospitalaria de forma particular y aislada, no es hasta 1998 cuando se firma un convenio entre Ministerio de Educación y Cultura, el Ministerio de Sanidad y Consumo y el Instituto Nacional de la Salud que establezca medidas concretas que garanticen que no exista solución de continuidad en la escolarización de los alumnos que o bien por una hospitalización prolongada o bien por las consecuencias de su enfermedad o su tratamiento no pueden asistir a sus colegios de referencia. Habían pasado 12 años desde la redacción de la Carta Europea de los Derechos del Niño Hospitalizado ${ }^{(1)}$ por el Parlamento Europeo (1986) y 9 desde la Convención sobre los Derechos del Niño ${ }^{(2)}$, tratado internacional firmado en el seno de las Naciones Unidas (1989). La garantía institucional a la educación de los niños enfermos existe en España, por tanto, desde hace dos décadas.

El mencionado Convenio entre el Ministerio de Educación y Cultura, el Ministerio de Sanidad y Consumo y el Instituto Nacional de la Salud para la atención educativa a los niños hospitalizados de 1998 expone en su cláusula primera: 
El presente Convenio tiene como objeto, en el ámbito territorial de gestión común del Instituto Nacional de la Salud y de Ministerio de Educación y Cultura, desarrollar un programa de atención educativa dirigido a la población hospitalizada en edad escolar obligatoria, que permita la continuidad de su proceso de escolaridad, favorezca su promoción académica y facilite, al tiempo, un marco educativo en los hospitales próximo a las necesidades psicosociales y afectivas de los niños hospitalizados. ${ }^{(3)}$

La única referencia al cuidado y curación de los niños resulta tan indefinida («facilite [...] un marco educativo [...] próximo a las necesidades psicosociales y afectivas de los niños hospitalizados») que roza lo tautológico. Es necesario comprobar si sus antecedentes son más concretos al respecto. En la Carta Europea de los Derechos del Niño Hospitalizado del Parlamento Europeo en 1986 se recoge, entre otros:

Derecho a proseguir su formación escolar durante su permanencia en el hospital, y a beneficiarse de las enseñanzas de los maestros y del material didáctico que las autoridades escolares pongan a su disposición, en particular en el caso de una hospitalización prolongada, con la condición de que dicha actividad no cause perjuicios a su bienestar y/o que no obstaculice los tratamientos que se siguen. ${ }^{(4)}$

Lo cual no resulta más alentador, pues considera la educación del niño un potencial obstáculo para su tratamiento («no cause perjuicios») en lugar de considerarla parte integrante del tratamiento. Por su parte, en el primer párrafo del artículo 28 de la Convención sobre los Derechos del Niño de Unicef en 1989 se puede leer:

1. Los Estados Partes reconocen el derecho del niño a la educación y, a fin de que se pueda ejercer progresivamente y en condiciones de igualdad de oportunidades ese derecho, deberán en particular:

a) Implantar la enseñanza primaria obligatoria y gratuita para todos;

b) Fomentar el desarrollo, en sus distintas formas, de la enseñanza secundaria, [...] hacer que todos los niños dispongan de ella y tengan acceso a ella $[\ldots] ;^{(5)}$

Es decir, no hay referencia a los niños hospitalizados, mientras que en los artículos dedicados a la atención médica no hay referencia a la educación.

Con este panorama puede concluirse que la consideración de la educación como herramienta del cuidado y curación del niño enfermo o siquiera como parte integrante de su tratamiento no está en el origen normativo de las aulas hospitalarias.

Como se ha visto, las mencionadas declaraciones provienen de la intención de subsanar una carencia (la educación de niños enfermos), es decir, en negativo, y no desde la perspectiva que se presenta en el presente artículo: la educación como herramienta en la curación y cuidado de un niño, es decir, en positivo ${ }^{5}$.

5 «Negativo»y «positivo» se utilizan aquí en el sentido de tratar de reparar un defecto frente al de crear una mejora. 
En ese sentido, la perspectiva normativa bajo la que nacen las aulas hospitalarias es de mínimos y no de máximos, es decir, se consideran útiles desde un sentido académico pero no se las imbrica en el funcionamiento de las unidades sanitarias donde se localizan. De hecho, dicha localización obedece a la necesidad de atender a los niños que allí se encuentran, no a una imbricación funcional y operativa con las correspondientes unidades hospitalarias donde se hallan.

Esa ausencia no es coherente con el cambio de perspectiva sobre la salud que se había operado en la comunidad internacional durante el siglo xx. Una lectura de la Constitución de la Organización Mundial de la Salud ${ }^{(5)}$ indica que el concepto de salud había variado para dejar de considerarse una noción estrictamente física e incluir consideraciones psicosociales. En ella puede leerse que «La salud es un estado de completo bienestar físico, mental y social, y no solamente la ausencia de afecciones o enfermedades».

Este giro conceptual de las declaraciones institucionales tiene su correlato académico. Pueden citarse la perspectiva holística(6)(7), el modelo psicosocial de Engel $^{(8)}$, el modelo salutogénico de Antonovsky ${ }^{(9)}$ o la salud positiva de Scales y Leffert ${ }^{(10)}$ como ejemplos de inserción de lo psicosocial en los procesos de mantenimiento y/o restablecimiento de la salud.

En todos los modelos mencionados la educación de niños y adolescentes enfermos tiene un papel crucial en el tratamiento de niños y jóvenes enfermos. Según LópezIbor ${ }^{(11)}$, el objetivo de la oncopediatría es conseguir que se conviertan en «adultos sanos desde un punto de vista físico, psíquico, social y espiritual», no solo por la importancia sustantiva de evitar secuelas psicológicas sino también por la facultad que posee la educación de dotar a los alumnos de herramientas posibilitadoras que los capaciten para protagonizar una vida adulta verdaderamente humana y completa.

Por su parte, Lizasoáin ${ }^{(12)}$ afirma que «La finalidad de la pedagogía hospitalaria es la misma que la de toda educación, es decir, el desarrollo integral de la persona, el desarrollo máximo de todas sus capacidades incluso en la situación de enfermedad y hospitalización». Por muy evidente que parezca esta aseveración, ese «máximo» conlleva una exigencia tanto para los docentes hospitalarios como para los discentes. La propia Lizasoáin ${ }^{(13)}$ estima que «Uno de los errores más frecuentes de los educadores actuales es el de facilitar demasiado las cosas a los educandos. No hay nada que mate más la ilusión que recibir todo lo que uno desea sin que se le haya exigido el menor esfuerzo».

El presente trabajo, dadas su metodología eminentemente cualitativa y la unicidad de su caso de estudio, no pretende tanto ser una demostración de lo expuesto supra sino un ejemplo real de los mecanismos según los cuales puede operar una experiencia educativa concernida por y enfocada a lo esencial ${ }^{6}$.

De todo lo anterior puede concluirse que, mientras la literatura normativa recoge una voluntad de mínimos en lo referente a las aulas hospitalarias y por tanto a la educación de los niños y adolescentes enfermos, tanto la práctica médica como la educativa exigen plantear una reformulación de la función y el desempeño de dichas aulas hospitalarias. En primer lugar, su objetivo no puede circunscribirse únicamente a garantizar la continuidad académica de los alumnos enfermos; en segundo, la realidad cambiante del alumnado de aulas hospitalarias y sus circunstancias dibuja un escenario de permanente adaptación y alta exigencia que no permite fiar su actividad 
a protocolos preestablecidos y planificaciones rígidas, y que por lo tanto depende en gran medida de la cualificación, capacidades y responsabilidad del profesorado.

Esta afirmación no solo se basa en consideraciones teóricas, sino que es una conclusión que dimana de la experiencia diaria en el aula hospitalaria de una Unidad de Hematología y Oncología Pediátrica de Montepríncipe. Ejemplificar lo antedicho es el objetivo del presente artículo.

\section{El caso}

Para ello se presenta el caso de J., un alumno de 17 años diagnosticado de un osteosarcoma localizado que requiere un tratamiento complejo que incluye quimioterapia pre- y postoperatoria, una cirugía de alta complejidad e inmunoterapia.

La duración del tratamiento es de 15 meses aproximadamente. A continuación requiere controles cada 3 meses hasta su alta definitiva a los 10 años de finalizar el tratamiento.

Asimismo requiere largo tratamiento de fisioterapia y rehabilitación, y tiene una alta probabilidad de secuelas físicas.

La situación del alumno cuando el Aula Hospitalaria empezó a funcionar en su nueva etapa ${ }^{7}$ era de total inactividad. Dicha situación se puede concretar en los siguientes puntos:

- A sus 17 años no se había matriculado en el curso que le correspondería haber empezado en aquel momento (septiembre de 2012), es decir, $1 .^{\circ}$ de Bachillerato (pues había repetido $4 .^{\circ}$ de Primaria debido a un cambio a un centro con un nivel más alto según él mismo refería).

- El alumno estaba totalmente apático, pasaba las horas ensimismado y lo único con lo que interactuaba era su tableta. No mostraba interés por ninguna de las consolas de videojuegos que estaban a su disposición. En el informe de seguimiento del 23 de julio de 2012 consta «No dolor. No fiebre. Aburrido».

- Además de no estar matriculado, tampoco tenía pensado hacer un repaso del currículo de Secundaria. Sus proyectos se limitaban a «sacarse el carné de conducir», actividad que tampoco realizaba en ese momento.

- El año anterior la experiencia del alumno con el Aula Hospitalaria en su primera etapa había sido negativa, según se inferiría de comentarios posteriores.

- El alumno se encontraba en medio de su tratamiento, con continuos ingresos hospitalarios.

- Había rechazado sucesivos acercamientos por parte del personal de la unidad.

En tales circunstancias, estaba claro que el primer contacto que se realizara por parte del nuevo profesorado sería crucial para el posterior desarrollo de los acontecimientos, sobre todo con vistas a acelerar el proceso de incorporación de J. al colegio.

7 El Aula Hospitalaria había funcionado el año anterior bajo la responsabilidad de un colegio de la zona, es decir, sin estar plenamente integrada en la Unidad de Oncopediatría. En el referido curso 2012/2103 iniciaba su andadura integrada en el equipo de psicosocial de la Unidad, y con nuevo profesorado. 
En una primera entrevista se le preguntó por su propósito, enfocando la propia Aula Hospitalaria como un posible elemento de ayuda si finalmente decidía cursar Bachillerato. En realidad nunca nos planteamos otra opción, y además descubrimos que la intención de J. era precisamente esa.

De lo que se trataba era de conseguir una rutina de trabajo en el Aula, de manera que aunque no estuviera matriculado en ningún centro y no tuviera que asistir todos los días al hospital por su tratamiento, sí acudiera al Aula Hospitalaria a realizar un trabajo tutelado y regular. La hipótesis era que, si J. percibía interés en los aspectos formativos de su situación por parte del profesorado, él mismo comenzaría a tomar interés en su aprendizaje.

A continuación se desarrollan los resultados del trabajo en cuanto a lo realizado con el alumno:

\subsection{Primer trimestre}

Por lo mencionado anteriormente se le propuso acudir al Aula Hospitalaria los días que tuviera que venir al hospital a causa del tratamiento, lo cual se consiguió desde el principio con cierta regularidad. Una vez constatamos que el nivel del alumno respecto a los contenidos de Secundaria en la asignatura de Matemáticas estaba lejos de cumplir unos mínimos, y ante el peligro de que un año de inactividad pudiera empeorar las cosas, decidimos centrarnos en repasar y asentar unos mínimos que le facilitaran el aprovechamiento del currículo de Bachillerato.

En ese sentido, se optó por repasar algunos conceptos y herramientas básicas de cálculo (números reales, fracciones, potencias, raíces y logaritmos, cálculo mental) y de álgebra (polinomios [factorización y productos notables], ecuaciones de primer y segundo grado, y sistemas de dos ecuaciones con dos incógnitas) en los que los alumnos de Bachillerato suelen mostrar lagunas importantes. Dadas las posibilidades que ofrecía el hecho de que J. no estuviera sujeto al ritmo de una programación temporal estricta, la estrategia seguida fue la de demorarnos en los contenidos hasta conseguir una cierta mecanización e interiorización de los mismos. El inicio no fue fácil, pues en todo momento se mantuvo una exigencia con respecto a su trabajo personal a la que el alumno no había estado acostumbrado. Esos parámetros de exigencia, además, se fueron elevando, a lo que el alumno respondió positivamente.

Otra de las facetas a las que se prestaron más atención fue el problema postural de J., tanto cuando se mantenía sentado en la silla de ruedas como cuando comenzó a caminar con muletas. Que estuviera más erguido y por tanto los demás lo vieran así generó un círculo virtuoso: al dejar de ser percibido por el resto de la Unidad como un adolescente introvertido, la interacción creció y el verdadero J. salió a la luz y fue conocido. Esto es una prueba de las consecuencias de la enfermedad, a menudo consideradas secundarias o inevitables — si no directamente ignoradas - pero que el equipo de psicosocial puede aliviar o solucionar.

Durante este periodo se tanteó la posibilidad (a falta de la autorización administrativa) de matricución a distancia para cursar $1 .^{\circ}$ de Bachillerato. Se desestimó esa opción a causa de su nivel, pero sobre todo de su actitud, que no era negativa en cuanto al comportamiento, pero sí todavía de cierta dejadez e indolencia. 


\subsection{Segundo trimestre}

Tras el primer trimestre se había conseguido muy poco en cuanto al aprendizaje de contenidos, pero mucho en lo referente a disciplina en la asistencia y en la ejecución de tareas. De hecho, durante el segundo trimestre J. comenzó a venir por iniciativa propia al colegio los días en que no tenía tratamiento, teniendo que vencer incluso para ello cierta resistencia familiar (por motivos de transporte).

Durante este segundo trimestre se produjo un cambio de ritmo en su aprendizaje. Ya se habían ampliado las clases al área de Lengua, y este segundo trimestre se hizo también con Filosofía. Nuestra intención era la de no aburrir al alumno repitiendo machaconamente los mismos contenidos, aunque en realidad sí se mantuvo como meta que J. dominara conceptos básicos en lugar de pasar por encima de la totalidad del temario sin que se consiguiera interiorizar ni mecanizar concepto ni procedimiento alguno.

Respecto a Lengua Castellana y Literatura, el alumno demostró enseguida tener muchos menos problemas que en Matemáticas, lo que se utilizó como refuerzo positivo tras la elevada exigencia mantenida durante el principio de curso (como se verá más abajo).

\subsection{Tercer trimestre}

El tercer trimestre no pudo ser presencial en su integridad, pues el 7 de mayo de 2013 el alumno terminó el tratamiento de quimioterapia y volvió a su ciudad de origen, pero continuó la atención por parte del aula por medios virtuales.

Durante este tercer trimestre, y gracias a la adquisición de los contenidos mínimos en Lengua y Matemáticas, iniciamos un trabajo de Historia que consistió en la realización de un eje cronológico a lo largo de una de las paredes del aula. El trabajo resultó motivador para J., quien mostró gran interés en su elaboración y en el seguimiento del mismo una vez hubo vuelto a su ciudad a mediados de mayo.

Por otra parte, se realizó un repaso de los contenidos estudiados a lo largo del curso, lo que permitió comprobar que gracias a la repetición se habían interiorizado las cuestiones más relevantes del álgebra y el cálculo (en el área de Matemáticas) y de sintaxis, comprensión lectora, figuras retóricas, comentario de texto y rudimentos de literatura medieval (en el área de Lengua).

\subsection{La exigencia}

Una vez el alumno comenzó a acudir a clase con cierta regularidad se pudo constatar que el reto era doble: por una parte, se trataba de afrontar las dificultades derivadas de su enfermedad, incluida la de no encontrarse matriculado en su curso de enseñanza reglada. Por otro lado, tras la prospección de nivel en las diferentes áreas (Lengua y Matemáticas, sobre todo), se pudo constatar que el nivel del alumno era muy pobre en el área de Matemáticas, pero también que no tenía hábito de estudio ni parecía haber seguido nunca rutinas de trabajo más o menos programado. Incidir y mejorar esos aspectos tan relevantes se convirtió en un objetivo tan importante como los meramente académicos. 
Con más detalle, el problema del alumno era múltiple:

1. Falta de capacidad de trabajo. No se le exigía desde su entorno ni él lo consideraba una necesidad.

2. Derivado de lo anterior, falta de nivel académico, percibida por el alumno con cierta autocomplacencia.

3. Dudas respecto a su propia capacidad, lo que podría tener con el tiempo efectos sobre su autoestima.

4. Problemas generados por su deslocalización de medio plazo, dado que el alumno se encontraba a cientos de kilómetros de su casa, su colegio y sus amigos.

5. Falta de responsabilidad sobre sus propios asuntos, tanto en la realización de tareas como en la adquisición y cuidado de su material.

Para contrarrestar estos condicionantes y tras comprobar que el alumno respondía bien a la disciplina — de hecho, se motivaba con la disciplina - se optó por establecer desde el principio una exigencia alta acompañada de signos externos que no le permitieran cuestionar las medidas tomadas. Los momentos en los que el alumno demostraba no haber trabajado lo suficiente o se mostraba remiso a pensar que podía llevar a cabo el aprendizaje las clases transcurrían en un clima de seriedad. Siempre se insistió en que él mismo estaba limitando su crecimiento, en que no era cierto que no pudiera alcanzar sus objetivos y en que lo único que le faltaba para hacerlo era esforzarse más. Se le hizo comprender que ni su enfermedad ni su tratamiento suponían un obstáculo real para su aprendizaje.

\subsection{La socialización}

La presencia en el colegio de otros alumnos, en concreto una alumna de $1 .^{\circ}$ de Bachillerato permitió realizar sesiones conjuntas en el trabajo de los contenidos comunes. La flexibilidad en la programación de J. permitió realizar estas adaptaciones.

La presencia de J. en el Aula coincidió con la definición paulatina y natural de un tiempo estipulado de recreo tal y como existe en los centros de referencia, es decir, un lapso en el que la tutela de los docentes es limitada y los alumnos interactúan mientras juegan, almuerzan o simplemente conversan. Ese tiempo libre puede parecer similar al que pasan en la unidad en función de su tratamiento y en horario no escolar (tardes, fines de semana), y sin embargo es totalmente distinto. El establecimiento de un horario académico convierte este recreo en una herramienta de normalización muy útil, dado que en ella los alumnos juegan (al aire libre cuando la meteorología lo permite), descansan y toman su almuerzo. A su vez, ese bocadillo es un claro exponente de lo que significa el recreo: mientras que la ingesta de alimentos en niños y adolescentes que reciben un tratamiento de quimioterapia puede ser un problema (falta de apetito, náuseas), cuando estos están inmersos en la dinámica escolar cotidiana toman ese bocadillo con total normalidad.

Hay que añadir que, para J., comprobar que otros alumnos estaban en una situación semejante y recibían una educación similar supuso un notable incentivo para continuar el trabajo. 


\section{Discusión}

Los siguientes son cambios observados en J. tras la actuación del Aula Hospitalaria:

1. Mejora del ánimo e implicación en la tarea, en general y respecto a los estudios en particular.

2. Mejoras posturales. Literalmente, el alumno comenzó a caminar más erguido.

3. Se percibió un cambio notable en la actitud de J. hacia el conocimiento en general, en concreto hacia las Humanidades. La posibilidad de trabajar sin la servidumbre de una programación (que por otra parte no estaba pensada para un alumno en su situación) permitió trabajar esos aspectos del currículo que más interés pudieran despertar en J..

4. El cambio más notorio, además de una adquisición de conocimientos apreciable, tuvo lugar en el aumento de la seguridad del alumno, aunque no tanto en su proactividad. Encontramos un alumno cabizbajo y poco expresivo y un año después se había convertido en un adolescente risueño y optimista, capaz de afrontar retos y con el firme propósito de estudiar una carrera universitaria.

5. En 2019 J. se encuentra cursando el último curso de un grado en Derecho.

\section{Conclusiones}

A menudo la visión existente sobre la asistencia a clase de un alumno con una enfermedad de estas características se acerca a «cubrir el expediente», «salir del paso» o incluso «así se distrae». Desgraciadamente dichas opiniones se basan a menudo en experiencias reales. Casos como el de J. demuestran, en cambio, que el papel de un aula hospitalaria puede $-\mathrm{y}$ debe - ir mucho más allá de la mera sustitución del colegio de referencia. La presencia de un personal especializado y experimentado posibilita que el aula hospitalaria sea una herramienta más para la curación del niño. En concreto, del presente estudio de caso pueden extraerse las siguientes conclusiones:

1. Una enfermedad como el cáncer afecta al ser humano en todas sus facetas, no solo físicamente sino también a su psicología y a su espíritu.

2. Dicha afectación no ocurre solo durante el tratamiento sino que con frecuencia lo acompaña el resto de su vida en forma de secuelas.

3. La curación del cáncer está cada vez más enfocada a eliminar o mitigar estas secuelas físicas y psicológicas, por lo que es importante atender a la psicología, educación y sociabilidad desde el momento del diagnóstico.

4. El aula hospitalaria debe ser un instrumento gracias al cual el alumno supere los retos psicológicos que el cáncer presenta. El aula hospitalaria debe ser una fuente de conocimiento en sentido amplio, es decir, un medio a través del cual se provea al alumno de herramientas para conocer y afrontar lo que le está ocurriendo, como parte de la vida y, en concreto, parte de $s u$ vida. La superación de retos, el crecimiento intelectual y la adquisición de una verdadera cultura demuestran aportar al alumno la suficiencia mental 
y física ${ }^{8}$ necesaria para afrontar el conjunto de cambios que la enfermedad comporta en todas las áreas de la persona.

5. Es obvio que las circunstancias de un alumno con cáncer no son las mismas que las de un alumno sano, y que estas circunstancias afectan a la forma en que encara al aprendizaje. Sin embargo, estas peculiaridades no tienen por qué lastrar su formación. La experiencia demuestra que la madurez de un alumno enfermo puede generar una capacidad inusitada para desarrollar su potencial intelectual, multiplicando las posibilidades de que el aprendizaje sea significativo y enriquecedor y lo ayude en su relación con su enfermedad y en su vida en general. Esta conclusión está basada en la observación de un gran número de casos y no en un optimismo voluntarista.

6. El caso concreto de J. demuestra la importancia de mantener un cierto grado de exigencia (teniendo siempre en cuenta que cada alumno es un caso particular y específico; no hay que olvidar que J. es un alumno varón de 17 años); a pesar de la tendencia natural a mostrarse condescendiente con alumnos enfermos, se ha comprobado que la exigencia, además de mejorar el rendimiento, ayuda a normalizar el día a día de los alumnos enfermos.

7. En la enseñanza de alumnos con cáncer tiene vital importancia la coordinación de todo el equipo de la unidad de oncología. Dado que la curación de niños y adolescentes ha de tener en cuenta los aspectos médicos, psicológicos, sociales y espirituales del enfermo, solo el trabajo conjunto de los diferentes profesionales de la unidad puede garantizar la atención al enfermo de forma global y verdaderamente humana.

8. Individualización de la enseñanza desde el punto de vista docente: no solo flexibilidad sino también estudio del caso concreto, independencia del currículo responsabilidad de los profesionales.

Es importante puntualizar que el caso de J. no tiene nada de extraordinario en cuanto a los resultados académicos que J. está consiguiendo en la Universidad: ese es el verdadero J.; el objetivo del equipo de psicosocial en particular y la Unidad en general fue mantener la normalidad en la vida de J. a largo plazo, para lo que es necesaria una atención profesionalizada, especializada y coordinada, es decir, el trabajo en equipo de toda la Unidad de Oncología Pediátrica.

En resumen: en una situación como el cáncer infantil y del adolescente es necesario que los profesionales sean proactivos, comprometidos y adaptativos para conseguir la normalidad en dos sentidos: primero, en el de conseguir que los alumnos lleguen a ser adultos sanos; segundo, en el de conseguir que el hecho de que un adulto que tuvo cáncer lleve una vida adulta sin secuelas psicosociales sea habitual y no excepcional.

\section{Referencias bibliográficas}

1. Parlamento europeo. Carta Europea de los Derechos del Niño Hospitalizado. 1986.

2. Unicef. Convención sobre los Derechos del Niño. 1989. 
3. BOE. Convenio entre el Ministerio de Educación y Cultura, el Ministerio de Sanidad y Consumo y el Instituto Nacional de la Salud para la atención educativa a los niños hospitalizados. 28 de julio de 1998; 25610:25612.

4. Parlamento europeo. Carta Europea de los Derechos del Niño Hospitalizado. 1986; 2.

5. Unicef. Convención sobre los Derechos del Niño. 1989; 22.

6. Organización Mundial de la Salud. Constitución de la Organización Mundial de la Salud. 1946; 1. [Acceso el 08/06/2020]. Disponible en: http://apps.who.int/gb/bd/PDF/bd47/SP/ constitucion-sp.pdf?ua $=1$

7. Laframboise H. Health Policy: breaking the problem down in more manageable segments. Can Med Assoc J 1973; 108; 388-93.

8. Lalonde M.. A new perspective on the health of Canadians. Ottawa, ON: Minister of Supply and Services Canada. 1974. Retrieved from Public Health Agency of Canada [Acceso el 08/06/2020]. Disponible en: http://www.phac-aspc.gc.ca/ph-sp/pdf/perspecteng.pdf

9. Engel GL. The need for a new medical model: a challenge for biomedicine. Science 1977; $196 ; 129-36$.

10. Antonovsky A. Health, Stress and Coping. San Francisco: Jossey-Bass, 1979.

11. Scales PC, Leffert N. Developmental assets: A synthesis of the scientific research on adolescent development. Minneapolis: Search Institute, 1999.

12. López-Ibor B. Aspectos médicos, psicológicos y sociales del cáncer infantil. Psicooncología 2009; 6: 281-4.

13. Lizasoáin O. Guía para la atención psicoeducativa del alumno enfermo. Madrid: Editorial Síntesis, 2016. p. 37.

14. Lizasoáin O. Guía para la atención psicoeducativa del alumno enfermo. Madrid: Editorial Síntesis, 2016.p. 122. 